\title{
Subcutaneous injection: learning from experience in other specialties
}

Debra Pollard, Steve Chaplin

Haemophilia treatment is characterised by intravenous infusions of clotting factor concentrates, with nurses frequently taking the lead role in administration, patient training and patient care. In recent years, a number of novel factor and non-factor-based therapies delivered by subcutaneous injection have been developed. These therapies are now undergoing clinical trials and will shortly be available in clinical practice. The coming era of at least some haemophilia treatments being delivered by subcutaneous injection clearly represents a significant change not only for patients (for whom they may be more convenient) but also for haemophilia nurse practice, particularly with respect to bleed and surgical management plans, and hence for nurse training and education. This review describes evidencebased guidance on subcutaneous injection technique and summarises the implications for nurses.

Keywords: Administration, Injection, Patient preference, Subcutaneous

n developed countries, most people with severe haemophilia currently receive prophylaxis with intravenous infusion of a clotting factor to prevent

bleeding episodes and reduce the risk of long-term complications. Inter-individual variation in the half-

\section{DEBRA POLLARD}

Lead Nurse Specialist, Katharine Dormandy Haemophilia \&

Thrombosis Centre, Royal Free London NHS Foundation

Trust, London, UK. Email: debra.pollard@enhs.net

STEVE CHAPLIN

Medical writer, UK. Email: stevechaplin504@gmail.com

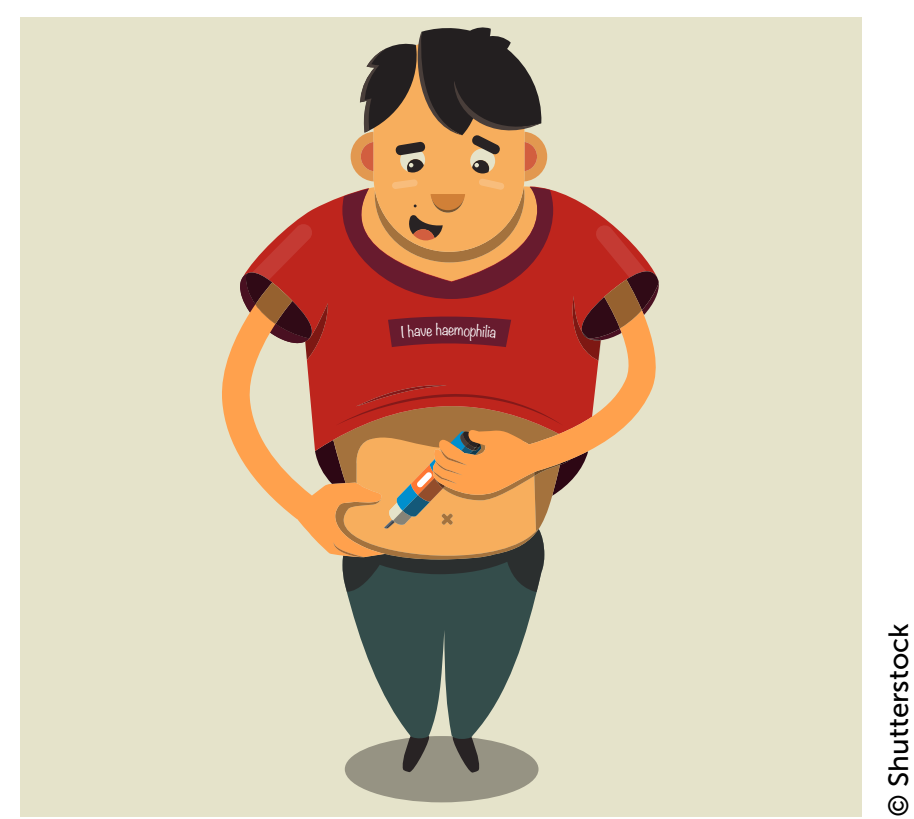

Image: A person with haemophilia administers their treatment using subcutaneous injection

life of replacement clotting factors means that the dose is tailored to each person's needs. Consequently, the frequency of infusion of factor VIII in people with haemophilia A varies between daily and every four days depending on individual pharmacokinetics and lifestyle ${ }^{[1]}$. Although there is inter-individual variability in the half-life achieved with replacement factor IX, this is generally longer than that of factor VIII; a onceor twice-weekly regimen is common ${ }^{[2]}$.

Novel agents now undergoing clinical trials offer a greater reduction in dose frequency. For example, emicizumab, a bi-specific monoclonal antibody that bridges activated factor IX and factor $X$ to restore the function of activated factor VIII, is administered once

This is an Open Access article distributed under the terms of the Creative Commons Attribution-NonCommercial-NoDerivs License (https://creativecommons.org/licenses/by-nc-nd/3.0/) which permits use and distribution in any medium, provided the original work is properly cited, the use is non-commercial, and no modifications or adaptations are made. Copyright is retained by the authors. 
UK nurses ready for subcutaneous therapy

In October to December 2017, Haemnet surveyed UK haemophilia nurses to establish their attitudes to and preparedness for subcutaneous therapy. The survey was conducted by a combination of telephone interviews and online questionnaire. In all, 72 haemophilia nurses from 41 NHS hospitals across the UK took part: $35 \%$ treated adults, $25 \%$ treated children and $40 \%$ treated both.

The survey suggested great variability in knowledge of hospital and departmental policies with regard to subcutaneous therapy. Nevertheless, nurses were overwhelmingly (98\%) confident in their ability to administer drugs by subcutaneous injection. Indeed, $85 \%$ of nurses said they regularly administered vaccinations and heparin subcutaneously. While two thirds (43/69) had witnessed local skin reactions, only $15 \%$ (10/69) had seen bleeding episodes following subcutaneous injection.

A wide variety of injection sites were recommended, and all respondents rotated injection sites on repeated injections. However, there was less agreement on other aspects of technique:

- $90 \%$ (65/72) "pinched an inch" but 10\% (7/72) did not

- $70 \%(50 / 72)$ cleaned the skin first but 30\% (22/72) did not

- $70 \%(50 / 71)$ pressed the skin but 30\% (21/71) did not

- $15 \%(11 / 72)$ rubbed the skin but $85 \%(61 / 72)$ did not

- 50\% (36/72) aimed for a 45-degree angle of entry while 30\% (22/72) aimed for 90 degrees and 20\% (14/72) expressed a variety of opinions.

There was general agreement that the fewer the number of injections per treatment the better, and that the maximum volume acceptable for a single injection should be $2 \mathrm{ml}$, but less for children.

Feedback from participants indicated that formal guidance on injection technique would be useful. Nevertheless, $97 \%$ of respondents were mostly or very confident in their ability to teach subcutaneous injection techniques to parents/patients, at broadly similar ages to those at which they would teach patients about intravenous prophylaxis. Written comments covered a range of views, from "I imagine a lot of patients will find it easier than IV" to "Sub-cut may be technically easier to administer but may actually be more painful for patients, so may not be as popular with patients as we imagine!"

weekly ${ }^{[4]}$; and fitusiran, an investigational RNA inference (RNAi) therapy that targets antithrombin, is administered monthly ${ }^{[5]}$. Products administered by subcutaneous injection currently in late-stage trials potentially herald a transformation in the delivery of haemophilia treatment.

Gaining competence in the administration of intravenous infusions requires significant training and depends largely on the quality of venous access, which is often poor in children, the elderly and those with significant comorbidities. Administration can be a considerable inconvenience for people with haemophilia and their carers, and some parents say the procedure is stressful for their children ${ }^{[6-8]}$. The latest generation of haemophilia treatments therefore offers less frequent injections by a route that might be expected to be more acceptable, with fewer complications and less impact on daily living.
This has implications for people with haemophilia, their carers and families, but also nurses and other health professionals. Among these is the need to raise knowledge among haemophilia nurses about best practice in the use of subcutaneous injections.

\section{Haemnet survey of haemophilia nurses}

In 2017, Haemnet carried out a survey involving 72 haemophilia nurses in 41 hospitals in the UK (data unpublished) - a detailed summary of the survey results is provided in the panel above. About one third cared solely for adults and one quarter solely for children. About half said their NHS Trust had a policy for subcutaneous injection and a quarter said they had a departmental policy. Almost all nurses said they were mostly or very confident about administering subcutaneous injections and $85 \%$ already administered other drugs by this route. 
There were differences in technique (e.g. angle of injection, rubbing the site) though these could not be correlated with the existence of a local policy. Feedback from participants indicated that formal guidance on injection technique would be useful.

\section{Guidance}

Guidelines on injection technique published by public institutions (e.g. health service or academic organisations) and professional groups often refer to, or are based on, the recommendations of the Safe Injection Global Network (SIGN). This voluntary coalition of stakeholders, formed to promote 'safe and appropriate use of injections throughout the world', is hosted by the World Health Organisation (WHO). Its publication, WHO best practices for injections and related procedures toolkit, is primarily concerned with policy development and infection control, in particular blood-borne viruses, but also offers advice on best practices for injection, summarising the general principles applicable to injection by all routes ${ }^{[9]}$.

Reflecting the prevalence of diabetes, much of the research into optimal subcutaneous injection technique has been for the administration of insulin. Evidence-based guidelines have been published by the Forum for Injection Technique and Therapy (FITTER), an international collaboration of clinicians, and the Forum for Injection Technique (FIT), a UK initiative led by diabetes specialist nurses and now involving many countries ${ }^{[10,11]}$. No other recent national guidelines about subcutaneous injection technique relevant to UK clinical practice were identified. There is, for example, no national guideline for the subcutaneous administration of heparin (Thrombosis UK, personal communication 2018). There are, however, many guidelines developed by NHS trusts for local use, such as those in the Royal Marsden Manual of Clinical Nursing Procedures ${ }^{[12]}$, that follow the principles set out in FIT.

FIT guidance is condensed into diabetes-specific 'golden rules' that could readily be adapted to the management of any condition requiring treatment by subcutaneous injection. FIT has also published guidance on the disposal of sharps and needlestick injury prevention, based on the EU Directive 2010/32 [13]. This summarises the regulatory requirements, actions to take in the event of a needlestick injury and the results of the FITTER survey of injuries involving nurses giving injections to patients with diabetes in a hospital setting ${ }^{[14]}$. Similar guidance has been published by the Forum for Injection Technique and Therapy Expert Recommendations, India ${ }^{[15]}$
The FIT golden rules cover psychological issues associated with treatment and administration; injection techniques for adults, young people and children; avoiding needlestick injuries; and sharps disposal. They provide a sound evidence-based model that can be adapted to the needs of people with haemophilia, carers and nurses, in particular because they address the needs of children and young people. These rules specifically address the issues arising from insulin therapy and the pharmaceutical aspects of insulin injection; their application in the haemophilia setting will need to take into account the properties of the novel agents and the different risks associated with the treatment of haemophilia. There is also a need to ensure compatibility with the current injection policies of NHS organisations.

A 2014 evidence-based review of subcutaneous injection technique drew extensively on the FIT guidance and noted several differences between recommendations for subcutaneous injection of insulin and heparin ${ }^{[16]}$. These were related to device-specific instructions (e.g. the air bubble in heparin prefilled syringes should be left in place to aid complete expulsion of the solution) and the exclusive use of the abdominal wall for injecting heparin (alternating sides) and are indicative of the changes that need to be made when developing injection guidance for other indications.

Advice on subcutaneous administration is normally provided in the literature supplied with a medicine. For example, emicizumab solution for injection (Hemlibra) is formulated as a solution for injection that needs to be drawn into a $1 \mathrm{ml}$ syringe for injection. Administration instructions are provided in the summary of product characteristics and the patient information leaflet. They include advice on the injection site (abdomen, the upper outer arms and the thighs are recommended but clinicians and carers may also use the upper outer arm); varying the injection site; avoiding sites that are red, bruised, tender or hard or areas where there are moles or scars; and not using the same site for injecting other medicines. Similar information may not be available for older medicines such as desmopressin. DDAVP is not licensed for subcutaneous injection for the management of haemophilia or von Willebrand's disease, therefore the manufacturer provides no information; no specific advice is provided with the Octim product. However, nurses have been using this treatment for many years, apparently without difficulty.

In summary, detailed guidance (national and local) on general strategies for subcutaneous injection technique is available and established in clinical practice. The precise steps required for particular 


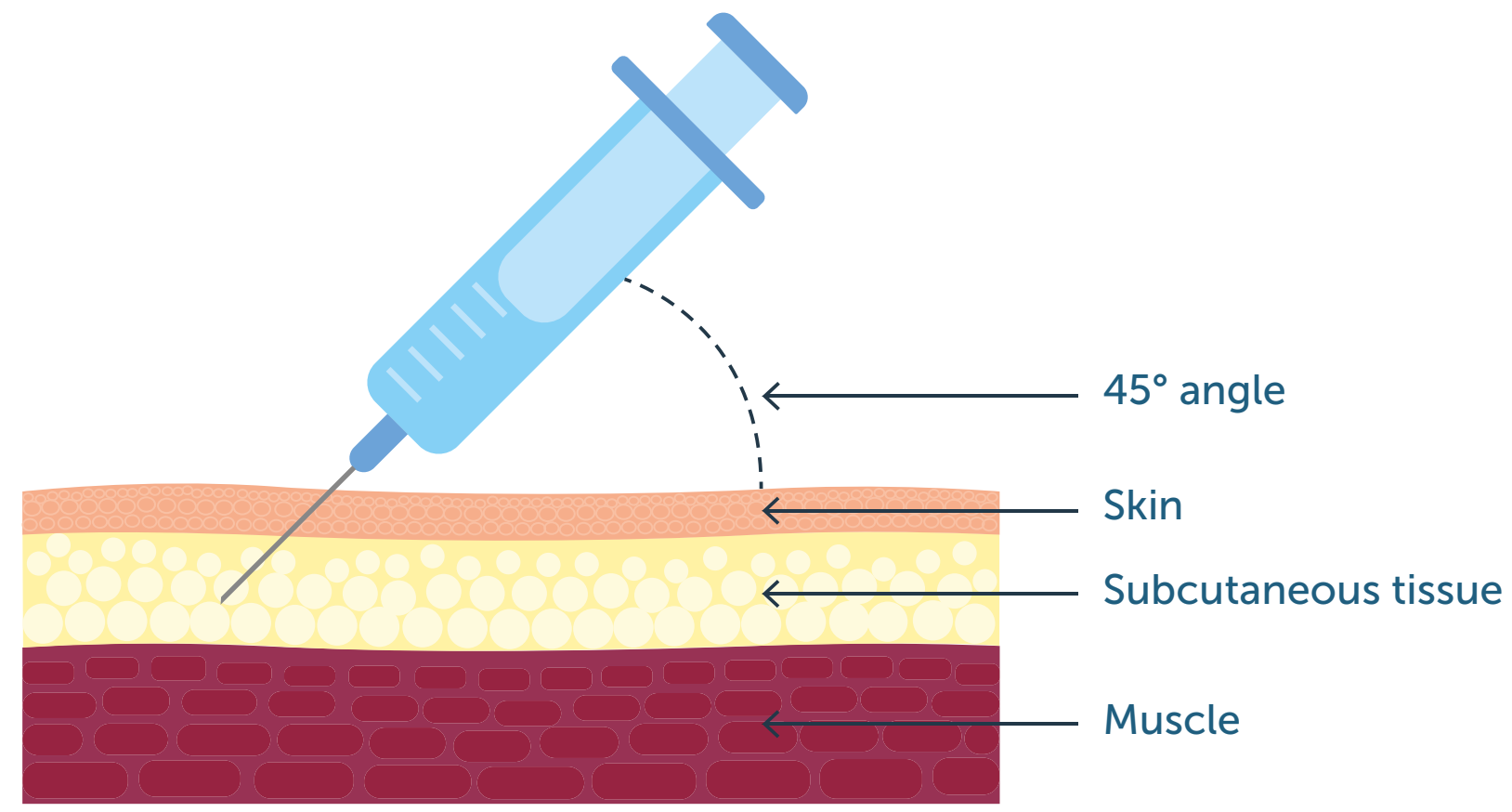

Figure 1: Subcutaneous injection should deliver treatment into the subcutaneous tissue beneath the skin, avoiding the underlying muscle. As a general recommendation, the needle should be inserted at a 45 -degree angle

medicines are influenced by their pharmacological properties and are not necessarily transferrable. The introduction of new haemophilia treatments should therefore be supported by practical training in administration technique for nurses.

\section{Injection technique}

The purpose of subcutaneous administration is to deliver the dose into the subcutaneous layer of the skin, where it forms a depot that is absorbed into the circulation at a predictable rate (see figure 1). Accidental deposition into the underlying musculature, which has a richer blood supply, or more superficially into the dermis, would alter the rate and possibly the extent of absorption and may be less well tolerated. The thickness of the subcutaneous layer varies between sites and individuals: it is generally lower among children, young people, lean people and older people. Injection technique should therefore include steps to ensure the dose is injected into the target area:

- The recommendations in published guidance are not consistent and the choice of needle size and injection technique is determined for each individual by the practitioner administering the injection, according to the patient's weight and body type

- The depth of subcutaneous tissue should be assessed by pinching the skin; this determines the appropriate needle size and the angle for administration
- There is no definitive evidence to support lifting a skin fold to raise subcutaneous tissue from the underlying musculature, but it is common practice when using needles of $\geq 5 \mathrm{~mm}$ length. The needle should be inserted into the fold at 45 degrees (this is not recommended for insulin).

- Raising a skin fold can be difficult in neonates, extremely thin people $(\mathrm{BMI}<19)$ and frail elderly people because they are likely to have limited subcutaneous tissue

- By contrast, it would be considered safe to use a standard length 25-gauge needle at a 90-degree angle for an obese adult

- The skin should not be pinched so tightly it causes pain or blanching, and the adjacent skin should not be depressed

- The plunger should not be withdrawn before drug administration: this may cause local trauma and the risk of entering a blood vessel is considered very low

- The area should not be massaged after withdrawing the needle

- The injection site should be varied, adhering to guidance on recommended body areas to use

The injection needle recommended for administering emicizumab solution for injection (Hemlibra) is $26 \mathrm{G}$, preferably 9-13 $\mathrm{mm}$ in length. Injection pens typically utilise a short needle length $(4-5 \mathrm{~mm})$. It is presently unclear whether the new clotting factor products will be presented as prefilled pens, syringes or vials. 


\section{Patient preferences for route of administration}

Though the practical experience of subcutaneous injection in the management of diabetes is unparalleled, the injection schedule of up to four injections daily (plus frequent finger prick testing) is dissimilar to the less frequent dose regimens likely for haemophilia. Further, most patients with diabetes have not previously been treated with an intravenous preparation and then offered a subcutaneous alternative as a treatment choice. In recent years, subcutaneous injection has become a more frequent option for a range of drugs originally administered intravenously for disorders that require lifelong treatment. This corresponds more closely with the choices soon available to the haemophilia community.

Biological disease-modifying antirheumatic drugs (bDMARDs) have established a prominent role in the management of rheumatoid arthritis and other autoimmune disorders, such as inflammatory bowel disease. These agents were initially administered by IV infusion with a frequency of twice weekly, weekly, or every two or four weeks. Subcutaneous administration has also become available for several drugs used in other indications, including trastuzumab (breast cancer) and the interferons (multiple sclerosis). Interferons were also used extensively for the treatment of hepatitis and many older patients with haemophilia may therefore have experience of subcutaneous injections. However, the acceptability of weekly subcutaneous injections of interferons does not appear to have been reported in people with haemophilia; interferons may be associated with adverse effects on mood (e.g. depression, fatigue), which may be a confounding factor. Little or none of the published evidence about preference appears to involve the experiences of children or young people.

Studies have not demonstrated a clear preference for subcutaneous injection in patients with rheumatoid arthritis. Asked which they prefer, $46-85 \%$ of patients opt for familiarity and choose their current therapy, whether subcutaneous or IV administration [17-19] Those who favoured subcutaneous injection cited autonomy, less time in clinic and cost as factors, whereas those rejecting a switch from IV administration cited concern about loss of hospital supervision, losing social relationships with other patients at the hospital, lower frequency of injection, fear of adverse events and - though this is not a consistent finding - fear of subcutaneous injections. In interviews, people with experience of treatment with a bDMARD contrasted the time required for IV infusion and the convenience of subcutaneous injection; those for whom travel to a hospital was a concern were more likely to favour subcutaneous injection ${ }^{[18]}$.

These trends are repeated in patients treated with biological agents for other indications. Interviews with patients receiving IV treatment for rheumatoid arthritis, Crohn's disease, ulcerative colitis, psoriatic arthritis, psoriasis or ankylosing spondylitis showed that $77 \%$ expressed very high satisfaction with their treatment and $82 \%$ preferred it to a subcutaneous alternative ${ }^{[20]}$. The most frequently cited reasons for preferring IV administration were not wanting to self-inject, less frequent dosing and preference for administration by a healthcare professional. When patients treated with IV infliximab or other bDMARDs administered subcutaneously were offered a hypothetical choice between the two, preference for current therapy was high ${ }^{[21]}$. However, preference for treatment at home was about twice as great as for clinic-based treatment

More patients with inflammatory bowel disease expressed a preference for IV infliximab infused every two months over subcutaneous adalimumab every two weeks, with about one third expressing no preference ${ }^{[22]}$. Reasons cited included distaste for self-injecting and the convenience of self-treatment at home. By contrast, a crossover randomised trial in women with primary breast cancer showed overwhelming preference for administering trastuzumab by subcutaneous injection (92\% vs 7\% for IV infusion) [23]. The reasons for preferring subcutaneous injection were time saving (80\%), less pain/discomfort/side-effects, ease of administration, convenience, problems with IV and less stress/anxiety. Reasons for preferring IV administration included fewer local reactions, psychological issues, perceived effectiveness and environment/staff ${ }^{[24]}$. In patients with multiple sclerosis treated with intramuscular (IM) interferon or subcutaneous alternatives, injection site reactions were reported in one to two thirds of patients using subcutaneous injections and $13 \%$ of those treated by IM injection ${ }^{[25]}$. Continuation rates were similar, but lack of effectiveness and injection site reactions were the most frequent reasons for switching or discontinuing treatment.

A systematic review published in 2015 found that the proportion of patients with a preference for subcutaneous administration was $44 \%-91 \%$ in four of six included studies; one study reported preference for IV administration and another found no difference ${ }^{[26]}$. Factors associated with subcutaneous preference were being able to have the treatment at home, time saving (e.g. travel time to the hospital), avoiding problems 
with IV administration or vein access, and reduced discomfort. One study found that subcutaneous injection offered no advantage for quality of life but a second reported that patients rated quality of life higher than during IV administration.

A review of 65 head-to-head studies of subcutaneous and intravenous administration of different drugs (not necessarily designed with patient preference as a primary endpoint) concluded that most comparisons favoured the subcutaneous route for trastuzumab, rituximab, anti-TNFs, bortezomib, amifostine, recombinant human granulocytemacrophage colony-stimulating factor, granulocyte colony-stimulating factor, recombinant interleukin-2, immunoglobulin, epoetin alfa, heparin and opioids. IV administration was superior only for ketamine, abatacept and vitamin $\mathrm{K}^{[27]}$. After defining safety and efficacy as the primary determinants of choice, the factors that influenced decision-making were identified as: patient's medical and physical characteristics; personal beliefs about safety; health literacy; perception of current morbidity; convenience and treatment history; socioeconomic status; issues arising from medication administration (site of injection, dose, frequency, administration time, indication, flexibility); and healthcare staff/institution-related factors.

\section{Modelling risk}

Subcutaneous injection is likely to be safer than intravenous infusion. Modelling the risks associated with each route of administration showed that subcutaneous injections was associated with less potential for errors such as dose calculation, preparation and package labelling, preparation of the access to the vein, pump infusion preparation and infusion monitoring ${ }^{[28]}$. This view was confirmed in a review of nursing perspectives on infliximab, in which the need for dosage calculations, aseptic preparation of infusion fluids, long infusion durations and extended medical staff input times, IV catheter placement and infusion-related reactions associated with intravenous infusion were contrasted with the simplicity and short administration time needed for subcutaneous administration ${ }^{[29]}$. There is good evidence that subcutaneous administration is associated with lower costs: the UK arm of a multinational study involving patients with lymphoma treated with rituximab found that active involvement of a health professional averaged 223 minutes per session with IV administration and 49 minutes with subcutaneous injection. Patients receiving the IV infusion spent a mean of 264 minutes in the treatment room compared with 70 minutes for the injection. Subcutaneous administration was associated with a mean reduction in staff costs of $£ 115.17$ per session ${ }^{[30]}$. Similar findings have been reported in the treatment of multiple myeloma with bortezomib [31].

\section{Summary}

1. Novel therapies will be administered by subcutaneous injection and have the potential to transform the treatment of haemophilia and service delivery

2. Comprehensive standards for subcutaneous injection technique have been established by the diabetes community; most of this guidance is directly applicable to the treatment of other disorders

3. The FIT golden rules provide a basis for developing guidance specific to the management of haemophilia

4. There is experience of switching from intravenous to subcutaneous administration in the management of several long-term disorders that require lifelong treatment. Little of this evidence is drawn from the experiences of children or young people.

5. The balance of published evidence appears to favour subcutaneous injections over intravenous administration, but studies directly reporting patient choice show that people differ in their preferences for route of administration, with a strong trend favouring whatever is their current therapy

6. Personal factors that favour switching to subcutaneous administration include convenience and the autonomy of self-treatment; factors favouring continued intravenous administration include fear of change, injection site reactions, supervision by health professionals and social contact with other patients

7. Subcutaneous injection offers savings in staff time and patient time compared with intravenous infusion

\section{Acknowledgements}

This article was commissioned as part of a Haemnet project funded by Roche Products and Chugai Pharma. The authors have advised no additional interests that might be perceived as posing a conflict or bias.

This article does not contain any studies involving human participants or animals performed by any of the authors.

\section{REFERENCES}

1. Richards M, Williams M, Chalmers E, et al. A United Kingdom Haemophilia Centre Doctors' Organization guideline approved by the British Committee for Standards in Haematology: guideline on the use of prophylactic factor VIII concentrate in children and adults with severe haemophilia A. Br J Haematol 2010; 149: 498-507. 
2. Berntorp E, Dolan G, Hay C, et al. European retrospective study of real-life haemophilia treatment. Haemophilia 2017; 23: 105-14.

3. Collins $P$, Chalmers $E$, Chowdary $P$, et al. The use of enhanced half-life coagulation factor concentrates in routine clinical practice: guidance from UKHCDO. Haemophilia 2016; 22: 487-98

4. Oldenburg J, Mahlangu JN, Kim B, et al. Emicizumab prophylaxis in hemophilia A with inhibitors. N Engl J Med 2017; 377: 809-18.

5. Pasi KJ, Rangarajan S, Georgiev P, et al. Targeting of antithrombin in hemophilia A or B with RNAi therapy. N Engl J Med 2017; 377: 819-28.

6. Hacker MR, Geraghty S, Manco-Johnson M. Barriers to compliance with prophylaxis therapy in haemophilia. Haemophilia 2001; 7: 392-6.

7. Shaw D, Riley GA. The impact on parents of developments in the care of children with bleeding disorders. Haemophilia 2008; 14: 65-7.

8. Furmedge J, Lima S, Monagle P, et al. 'I don't want to hurt him.' Parents' experiences of learning to administer clotting factor to their child. Haemophilia 2013; 19: 206-11.

9. World Health Organisation. WHO best practices for injections and related procedures toolkit. 2010. Available from www.ncbi.nlm.nih.gov/books/NBK138491 (accessed 16 April 2018)

10. Frid $A H$, Kreugel $G$, Grassi $G$, et al. New insulin delivery recommendations. Mayo Clin Proc 2016; 91: 1231-55.

11. FIT UK Forum for Injection Technique UK. The UK Injection and Infusion Technique Recommendations, 4th edn. October 2016. Available from http://www.fit4diabetes.com/ files/4514/7946/3482/FIT_UK_Recommendations_4th_ Edition.pdf (accessed 16 April 2018).

12. The Royal Marsden NHS Foundation Trust. The Royal Marsden Manual of Clinical Nursing Procedures, 9th edn, 2015. Available from http://www.rmmonline.co.uk/ (accessed 16 April 2018).

13. FIT4Safety. Injection Safety in UK and Ireland; Safety of Sharps in Diabetes Recommendations, 1st edn. 2012. Available from http://www. fit4diabetes.com/ files/1413/4727/6994/BD4224_FIT_Safety_STG07_AW2 PP.pdf (accessed 16 April 2018).

14. Costigliola V, Frid A, Letondeur, $C$ et al. Needlestick injuries in European nurses in diabetes. Diabetes Metab 2012; 38 Suppl 1: S9-14.

15. Tandon N, Kalra S, Balhara YPS, et al. Forum for injection technique and therapy expert recommendations, India: the Indian recommendations for best practice in insulin injection technique, 2017. Indian J Endocrinol Metab 2017; 21: 600-17.

16. Ogston-Tuck S. Subcutaneous injection technique: an evidence-based approach. Nurs Stand 2014; 29: 53-8.

17. Desplats $M$, Pascart $T$, Jelin $G$, et al. Are abatacept and tocilizumab intravenous users willing to switch for the subcutaneous route of administration? A questionnaire-based study. Clin Rheumatol 2017; 36: 1395-1400.
18. Chilton F, Collett RA. Treatment choices, preferences and decision-making by patients with rheumatoid arthritis. Musculoskelet Care 2008; 6: 1-14.

19. Huynh TK, Ostergaard A, Egsmose C, et al. Preferences of patients and health professionals for route and frequency of administration of biologic agents in the treatment of rheumatoid arthritis. Patient Prefer Adherence 2014; 8: 93-9.

20. Bolge SC, Eldridge HM, Lofland JH, et al. Patient experience with intravenous biologic therapies for ankylosing spondylitis, Crohn's disease, psoriatic arthritis, psoriasis, rheumatoid arthritis, and ulcerative colitis. Patient Prefer Adherence 2017; 11: 661-9.

21. Sylwestrzak G, Liu J, Stephenson JJ, et al. Considering patient preferences when selecting anti-tumor necrosis factor therapeutic options. Am Health Drug Benefits 2014; 7: 71-81.

22. Allen PB, Lindsay $H$, Tham TC. How do patients with inflammatory bowel disease want their biological therapy administered? BMC Gastroenterol 2010; 10:1. doi: 10.1186/1471-230X-10-1.

23. Pivot X, Gligorov J, Müller V, et al. Preference for subcutaneous or intravenous administration of trastuzumab in patients with HER2-positive early breast cancer (PrefHer): an open-label randomised study. Lancet Oncol 2013; 14: 962-70.

24. Jackisch C, Müller V, Dall P, et al. Subcutaneous trastuzumab for HER2-positive breast cancer - evidence and practical experience in 7 German centers. Geburtshilfe Frauenheilkd 2015; 75: 566-73.

25. Beer K, Müller M, Hew-Winzeler AM, et al. The prevalence of injection-site reactions with disease-modifying therapies and their effect on adherence in patients with multiple sclerosis: an observational study. BMC Neurol 2011; 11: 144.

26. Stoner KL, Harder H, Fallowfield LJ, et al. Intravenous versus subcutaneous drug administration. Which do patients prefer? A systematic review. Patient 2015; 8: 145-53.

27. Jin JF, Zhu LL, Chen M, et al. The optimal choice of medication administration route regarding intravenous, intramuscular, and subcutaneous injection. Patient Prefer Adherence 2015; 9: 923-42

28. Ponzetti C, Canciani M, Farina M, et al. Administrative risk quantification of subcutaneous and intravenous therapies in Italian centers utilizing the Failure Mode and Effects Analysis approach. Clinicoecon Outcomes Res 2016; 8: 353-9.

29. Carlson J, Cox K, Bedwell K, et al. Rituximab for subcutaneous delivery: clinical management principles from a nursing perspective. Int J Nurs Pract 2015; 21 Suppl 3: 1-13.

30. Rule S, Collins GP, Samanta K. Subcutaneous vs intravenous rituximab in patients with non-Hodgkin lymphoma: a time and motion study in the United Kingdom. J Med Econ 2014; 17: 459-68.

31. Barbee MS, Harvey RD, Lonial S, et al. Subcutaneous versus intravenous bortezomib: efficiency practice variables and patient preferences. Ann Pharmacother 2013; 47: 1136-42.

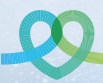

The Journal of Haemophilia Practice
An open-access journal for sharing experience in the care of people with bleeding disorders 